\title{
Towards Detection of Single Rare-Earth-Ions in a Nanophotonic Resonator
}

\author{
Tian Zhong, Jonathan Kindem, Evan Miyazono, and Andrei Faraon \\ T. J. Watson Laboratory of Applied Physics, California Institute of Technology, 1200 E California Blvd, Pasadena, CA, 91125, USA \\ tzhong@,caltech.edu
}

\begin{abstract}
We report a scheme for detecting single rare-earth-ions coupled to an Yttrium Orthosilicate (YSO) nanophotonic resonator, which could enable precise optical addressing of individual ions as single qubits for quantum information applications.

(C)2015 Optical Society of America

OCIS codes: (270.0270) Quantum optics; (270.5585) Quantum information and processing; (270.5580) Quantum electrodynamics
\end{abstract}

The narrow optical transitions, long coherence times and strong dipole-dipole interactions of rare-earth-ions (REI) in crystals make them attractive for a number of quantum information applications, including quantum memory and quantum logic gates [1]. A single REI is particularly interesting as it can serve as a single qubit, allowing direct optical addressing and control [2]. However, the weak emission associated the long excited-state lifetimes has restricted previous studies mostly to large ensembles of ions. The investigation at a few ions or single ion level has been challenging, primarily due to their faint florescence and very narrow spectral widths $(\sim \mathrm{kHz})$. Here, we report a scheme to detect single rare-earth-ions by measuring reduced cavity transmission when a REI is coupled to the cavity. Purcell-enhanced coupling to the cavity results in increased single ion emission, as well as a distinct narrow dip in the transmitted intensity - a coherent effect by which a single coupled ion significantly changes the cavity transmission. Using a $0.2 \%$ Neodymium (Nd) doped Yttrium Orthosilicate (YSO) nanophotonic cavity, we observed this coherent effect due to a small ensemble of ions, on the order of 10 ions per homogeneous linewidth. The same measurement in a $0.003 \%$ doped $\mathrm{Nd}$ :YSO nano-cavity will yield signals expectedly from a single $\mathrm{Nd}$ ion. Our detection scheme is naturally suited for addressing single REI emitters on-chip, and thus provides an avenue towards an integrated quantum photonic network based on REIs.

The detection scheme is illustrated in Fig. 1(a). An ensemble of inhomogeneously broadened REIs are embedded in a two-sided optical cavity. With a single ion-cavity coupling coefficient g, cavity decay rate $\kappa$, ion homogeneous linewidth $\Gamma_{h}$, and atomic density $N$ ions per $\Gamma_{h}$, the normalized cavity transmission under a weak coherent excitation at frequency $\omega$ is

$$
T(\omega)=\left(\frac{\kappa}{\kappa+N g^{2} /\left(i\left(\omega-\omega_{\text {ion }}\right)+\Gamma_{h}\right)}\right)^{2} .
$$

Typical cavity transmission as a function of frequency detuning $\omega-\omega_{\text {ion }}$ is plotted in Fig. 1 (b), assuming cavity parameters $Q=4000$, mode volume $V=1.6(\lambda / n)^{3}$, ion linewidth $\Gamma_{h}=4 \mathrm{kHz}$ and ion density $N=1$. The dip in the transmission is due to a single ion coupled to the cavity. This effect, previously referred to as dipole-induced transparency (DIT) [3], originates from destructive interference between the intra-cavity field and the coherently driven ion. The contrast of the dip is determined by the cooperativity of the ion-cavity coupling $g^{2} / \kappa \Gamma_{h}$. Complete extinction of transmission can be achieved using a nanophotonic cavity with a small mode volume and high $Q$ [4].

a

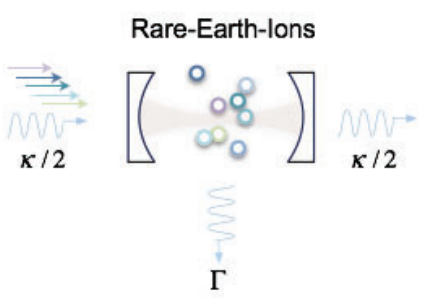

b

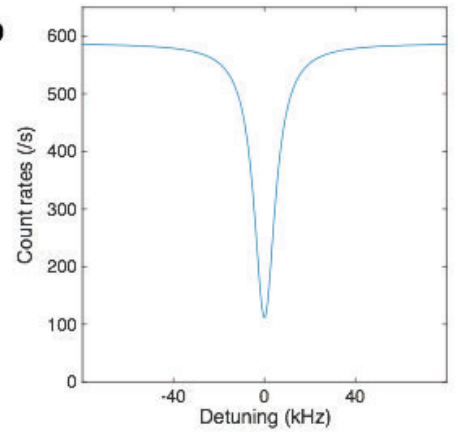

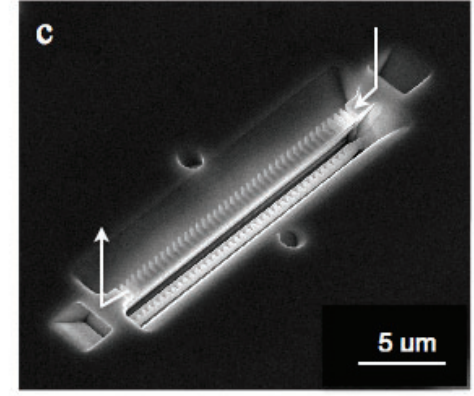

Fig. 1. (a) Schematics for detecting single REIs coupled to a two-side cavity. Cavity transmission is measured while laser frequency at the input (left) is scanned (denoted by varying colors). (b) Calculated transmission with a single ion coupled to the cavity. (c) Fabricated Nd:YSO nanophotonic resonator consisting of a nano-beam cavity and two end reflectors for vertical coupling. 
We fabricated nano-beam resonators in Nd:YSO using focused ion beam. For a $0.2 \%$ doped high-density $\mathrm{Nd}$ :YSO device, resonance of $\mathrm{Q}=4400$ and mode volume $V=1.6(\lambda / n)^{3}$ at the $883 \mathrm{~nm} \mathrm{Nd}$ transition was achieved. Inhomogeneous and homogeneous linewidths of the ions in the cavity were measured to be $16 \mathrm{GHz}$, and $100.6 \mathrm{kHz}$ at $3.5 \mathrm{~K}$ temperature, respectively, with applied magnetic field of $500 \mathrm{mT}$ at $30^{\circ}$ angle to $D_{2}$ axis. Vertical in/out coupling to the cavity via a confocal microscope was realized by two efficient reflectors, as shown in Fig.1 (c). Cavity transmission was scanned using attenuated Ti:Sapphire laser of $\sim 50 \mathrm{kHz}$ linewidth. The laser continuously scans a $25 \mathrm{GHz}$ bandwidth in $10 \mathrm{~s}$, while a high-sensitivity spectrometer registers the photon counts intermittently with an exposure time $0.01 \mathrm{~s}$ in each shot. Therefore one exposure corresponds to a spectral width of $25 \mathrm{MHz}$ scanned by the laser. Note that for this high-density device, dips due to different ions are superimposed, forming a continuous distribution that is related to the local ion density ( $\mathrm{N}$ ions per $\Gamma_{h}$ linewidth).

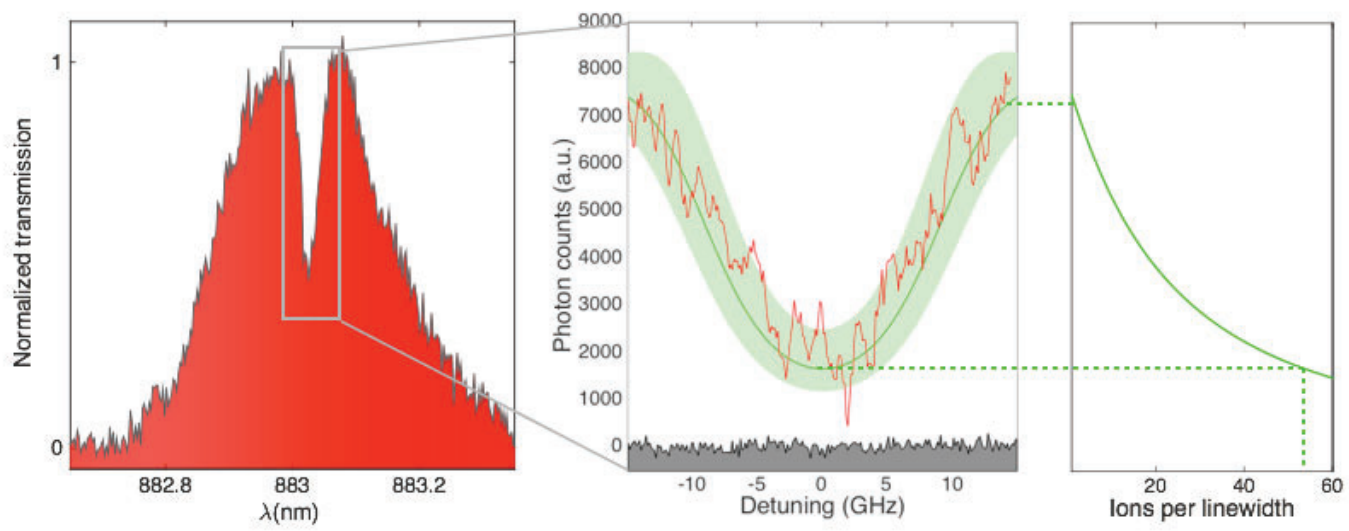

Fig. 2. Measured high-density Nd:YSO nano-cavity transmission with a broadband source (left panel), and an attenuated Ti:Sapphire laser (middle panel). Green line is the fit for the ion density based on Eq. (1). The green shade is the calculated uncertainty based on ion density shot noise.

Figure 2 plots the measured transmission against laser detuning, showing a DIT signal directly related to the ion spectral density. By fitting the data using Eq. (1) (green line), we deduced a peak ion density of $N \sim 53$ ions per $\Gamma_{h}$ linewidth. The green shade is the calculated uncertainty from the shot noise of ion density, which agrees well with measurement. Note that this atomic shot noise is significantly larger than the technical noise of the spectrometer (grey background). Additionally, we verified the data against signals taken at larger detunings $(>50$ $\mathrm{GHz}$ ) where no ion is present. The absence of large signal variations outside the ion inhomegeneous linewidth confirms the shot noise limited ion density observed in the middle panel of Fig. 2.

We also fabricated a $0.003 \%$ doped low-density Nd:YSO resonator with $\mathrm{Q}=3800$. In this device, $\Gamma_{h}$ was $\sim 8.8$ $\mathrm{kHz}$ with $100 \mathrm{mT}$ magnetic field, and the peak ion density was $\sim 0.2$ ion per $\Gamma_{h}$. By performing the same measurement before in this low-density device, we expect to see isolated dips due to individual Nd ions. To better resolve signals from single ions, it is necessary to use a frequency stabilized laser with linewidth $\sim 1 \mathrm{kHz}$. We custom built a fiber-based reference cavity of linewidth $1 \mathrm{MHz}$. Using Pound-Drever-Hall (PDH) locking, we were able to reduce the Ti:Sapph laser linewidth to $<10 \mathrm{kHz}$. With further improvement, we expect to achieve a laser linewidth of $1 \mathrm{kHz}$, with a long term drift less than $1 \mathrm{kHz} / \mathrm{s}$. This stabilized laser, combined with a slowly frequency sweeping acousto-optic modulator (AOM), could in principle resolve the single ion signal. Once identified, $\mathrm{g}^{(2)}$ correlation measurement will be followed to verify the emission from a single ion.

\section{References}

[1] C. Thiel, T. Böttger, and R. Cone, "Rare-earth-doped materials for applications in quantum information storage and signal processing," $J$. Luminesc. 131, 353-361 (2001).

[2] T. Utikal, E. Eichhammer, L. Petersen, A. Renn, S. Götzinger, and V. Sandoghdar, "Spectroscopic detection and state preparation of a single praseodymium ion in a crystal," Nat. Commun. 5, 3267 (2014).

[3] E. Waks and J. Vuckovic, "Dipole induced transparency in drop-fileter cavity-waveguide systems," Phys. Rev. Lett. 96, 153601 (2006).

[4] D. L. McAuslan and J. J. Longdell, "Cavity QED using rare-earth-metal-ion dopants in monolithic resonators: What you can do with a weak oscillator," Phys. Rev. A 80, 062307 (2009). 